Understanding the dramatic role of anomalous dispersion on the measurement of electron densities in plasmas using interferometers

Joseph Nilsen, Walter R. Johnson, Carlos A. Iglesias, James H. Scofield

July 22, 2005

SPIE International Symposium on Optics and Photonics San Diego, CA, United States July 31, 2005 through August 4, 2005 
This document was prepared as an account of work sponsored by an agency of the United States Government. Neither the United States Government nor the University of California nor any of their employees, makes any warranty, express or implied, or assumes any legal liability or responsibility for the accuracy, completeness, or usefulness of any information, apparatus, product, or process disclosed, or represents that its use would not infringe privately owned rights. Reference herein to any specific commercial product, process, or service by trade name, trademark, manufacturer, or otherwise, does not necessarily constitute or imply its endorsement, recommendation, or favoring by the United States Government or the University of California. The views and opinions of authors expressed herein do not necessarily state or reflect those of the United States Government or the University of California, and shall not be used for advertising or product endorsement purposes. 


\title{
Understanding the dramatic role of anomalous dispersion on the measurement of electron densities in plasmas using interferometers
}

\author{
Joseph Nilsen $^{1}$, Walter R. Johnson ${ }^{2}$, Carlos A. Iglesias ${ }^{1}$, and James H. Scofield ${ }^{1}$ \\ ${ }^{1}$ Lawrence Livermore National Laboratory, Livermore, CA 94551 \\ ${ }^{2}$ University of Notre Dame, Notre Dame, IN 46556
}

\begin{abstract}
For decades the electron density of plasmas has been measured using optical interferometers. With the availability of good X-ray laser sources in the last decade interferometers have been extended into the wavelength range $14-47 \mathrm{~nm}$, which has enabled researchers to probe even higher density plasmas. The data analysis assumes the index of refraction is due only to the free electrons, which makes the index less than one. Recent interferometer experiments in Al plasmas observed plasmas with index of refraction greater than one at $14 \mathrm{~nm}$ and brought into question the validity of the usual formula for calculating the index. In this paper we show how the anomalous dispersion from bound electrons can dominate the free electron contribution to the index of refraction in many plasmas and make the index greater than one or enhance the contribution to the index such that one would greatly overestimate the density of the plasma using interferometers. Using a new average-atom code we calculate the index of refraction in many plasmas at different temperatures for photon energies from 0 to $100 \mathrm{eV}$ and compare against calculations done with OPAL. We also present examples of other plasmas that may have index of refraction greater than one at X-ray laser energies. During the next decade X-ray free electron lasers and other X-ray sources will be available to probe a wider variety of plasmas at higher densities and shorter wavelengths so understanding the index of refraction in plasmas will be even more essential.
\end{abstract}

Keywords: X-ray laser, Interferometers; Index of refraction; Plasmas; Anomalous dispersion

\section{INTRODUCTION}

Since the early days of lasers optical interferometers have been used to measure the electron density of plasmas [1] using the assumption that the index of refraction of the plasma is due only to the free electrons [1-2]. This implies that the electron density of the plasma is directly proportional to the number of fringe shifts in the interferometer and that the index of refraction in the plasma is always less than one. Ten years ago, the first X-ray laser interferometer [3] was demonstrated at the Nova facility at Lawrence Livermore National Laboratory (LLNL) using the neon-like yttrium laser that operated at 15.5-nm. Over the last decade many X-ray laser interferometers [4-6], as well as a high order harmonic interferometer [7], have been built in the wavelength range of 14 to $72 \mathrm{~nm}$. All the experiments done with these sources assume only the free electrons contribute to the index of refraction. In the future, interferometers will be built using the VUV and X-ray free electron lasers, which will extend lasers to even shorter wavelengths [8]. 
In the last two years interferometer experiments [4,5] of Al plasmas observed fringe lines bend in the opposite direction than was expected, indicating that the index of refraction was greater than one. The traditional analysis of the experiments would indicate that the electron density was less than zero. Those experiments were done at the Advanced Photon Research Center at JAERI using the $13.9 \mathrm{~nm}$ Ni-like Ag laser [4] and at the COMET laser facility at LLNL using the $14.7 \mathrm{~nm} \mathrm{Ni-like} \mathrm{Pd} \mathrm{laser} \mathrm{[5].} \mathrm{Analysis} \mathrm{of} \mathrm{the} \mathrm{experiments} \mathrm{showed} \mathrm{that} \mathrm{the} \mathrm{anomalous} \mathrm{dispersion} \mathrm{from} \mathrm{the}$ resonance lines and absorption edges of the bound electrons have a large contribution to the index of refraction with the opposite sign as the free electrons and this explains how the index of refraction is greater than one in some Al plasmas [9]. A surprising result of the calculations is that the influence of the bound electrons on the index of refraction extends far from the absorption edges and resonance lines [9]. Resonance lines affect the index of refraction at photon energies located orders of magnitude further from the line centers than the corresponding line widths owing to the fact that they contribute through a dispersion integral.

The original analysis [9] of the index of refraction for $\mathrm{Al}$ plasmas was done at a single wavelength, $14.7 \mathrm{~nm}$, by combining individual calculations done for each iso-electronic sequence of Al. That analysis pointed out the need for a new tool that would let us calculate the index of refraction for any plasma at any wavelength. For many years the INFERNO average atom code has been used to calculate the distribution of levels and the absorption coefficient for plasma at a given temperature and density [10]. By modifying this code [11], we are now able to calculate the index of refraction for a wide range of plasma conditions. In this work we present results for Al plasmas from singly to many times ionized. We consider photon energies from 0 to $100 \mathrm{eV}(12.4 \mathrm{~nm})$ because this is the range of most X-ray laser sources. However the code can also be used for much higher energies. These calculations enable us to understand under what plasma conditions the free electron approximation is valid and gives us an estimate of the magnitude of the bound electron contribution. To validate the average atom code results for Al plasmas we compare with calculations done with the more detailed OPAL code [12-14].

We search for other materials that can be used to create plasmas with index of refraction greater than one at X-ray laser energies. We discuss how doubly-ionized $\mathrm{Ag}$ and $\mathrm{Sn}$ look to be promising candidates to use in interferometer experiments based on the Ne-like Ar X-ray laser at $26.44 \mathrm{eV}$. For triply-ionized Xe, we present calculations that show that this plasma will have an index of refraction greater than one at the energy of the Pd X-ray laser at $84.46 \mathrm{eV}$.

\section{ANALYSIS OF INTERFEROMETER EXPERIMENTS}

The traditional formula for the index of refraction of a plasma is $n=\left(1-\mathrm{N}_{\text {elec }} / \mathrm{N}_{\text {crit }}\right)^{1 / 2}$ where $\mathrm{N}_{\text {elec }}$ is the electron density of the plasma and $\mathrm{N}_{\text {crit }}$ is the plasma critical density. This assumes that only free electrons contribute to the index of refraction. At wavelength $\lambda, \mathrm{N}_{\text {crit }}=\pi /\left(\mathrm{r}_{0} \lambda^{2}\right)$ where $\mathrm{r}_{0}$ is the classical electron radius, $2.818 \times 10^{-13} \mathrm{~cm}$ [2]. In typical experiments the electron density is much less than the critical density so the formula above can be approximated by $\mathrm{n}=1-\left(\mathrm{N}_{\text {elec }} / 2 \mathrm{~N}_{\text {crit }}\right)$. For a plasma that is uniform over length $\mathrm{L}$ the number of fringe shifts observed in an interferometer equals $(1-n) L / \lambda$. For a non-uniform plasma one does a path length integral. The formula assumes that the interferometer is in a vacuum so that the index of refraction is one except for the probed plasma. The fringe shifts are referenced against a set of reference fringes in the absence of any plasma. Using the approximations described above, the number of fringe shifts equals $\left(\mathrm{N}_{\text {elec }} \mathrm{L}\right) /\left(\begin{array}{lll}2 & \lambda \mathrm{N}_{\text {crit }}\end{array}\right)$. When analyzing an experiment one counts how far the fringes have shifted compared with the reference fringes and converts this into electron density. For the $14.7 \mathrm{~nm}$ Pd X-ray laser the number of fringe shifts in the interferometer is $\left(\mathrm{N}_{\text {elec }} \mathrm{L}\right) /\left(1.5 \times 10^{19} \mathrm{~cm}^{-2}\right)$ and the critical density is $5.17 \times 10^{24} \mathrm{~cm}^{-3}$. From the anomalous results in the interferometer experiments [4,5] of the Al plasmas it is clear that the traditional technique used to analyze the interferometer experiments is incomplete and that the bound electrons have a significant contribution to the index of refraction for the Al plasmas. 
To understand the role of the bound electrons Ref. 9 calculated the continuum absorption cross-sections $\sigma$ for each ionization stage of $\mathrm{Al}$ using a Hartree-Slater code. The energies were adjusted to make certain the $\mathrm{L}_{3}$ edges for the $2 p$ electrons agreed with the experimentally measured edges for neutral, singly, doubly, and triply ionized Al. The contributions from the absorption lines were then added to the continuum absorption. For singly and doubly ionized $\mathrm{Al}$ the measured line positions and oscillator strengths from Refs. 15 and 16 for the lines below the L3 edges were used. For triply ionized Al the line positions and strengths from Ref. 17 were used. The total absorption coefficient $\alpha=$ $\mathrm{N}_{\text {ion }} \sigma=(4 \pi \beta) / \lambda$ where $\mathrm{N}_{\mathrm{ion}}$ is the ion density of the plasma and $\beta$ is the imaginary part of the complex index of refraction $n^{*}$ defined by $n^{*}=1-\delta-i \beta$. The real part of the index of refraction $n=1-\delta$. The Henke tables tabulates the dimensional-less optical constants $\mathrm{f}_{2}$ and $\mathrm{f}_{1}$ which are related to $\delta$ and $\beta$ by $\delta=\mathrm{f}_{1} \mathrm{~N}_{\text {ion }} /\left(2 \mathrm{~N}_{\text {crit }}\right)$ and $\beta=\mathrm{f}_{2} \mathrm{~N}_{\text {ion }} /\left(2 \mathrm{~N}_{\text {crit }}\right)$ [18]. From the total absorption cross-section $\sigma$ we determine the optical constant $f_{2}=\sigma /\left(2 \lambda r_{0}\right)$. We then derive the optical constant $f_{1}$ as a function of photon energy $E$ using the Kramers-Kronig dispersion relation [19]. This involves taking the principal value of the integral

$$
f_{1}(E)=Z_{n u c}+\frac{2}{\pi} P . V \cdot \int_{0}^{\infty} \frac{f_{2}(\varepsilon) \varepsilon d \varepsilon}{E^{2}-\varepsilon^{2}}
$$

where $Z_{\text {nuc }}$ is the atomic number of the element. This means we include the total number of bound and free electrons when calculating the dispersion relation. For example, $Z_{\mathrm{nuc}}=13$ for an $\mathrm{Al}$ plasma. For neutral materials the oscillator sum rules insure that $f_{1}$ goes to zero at zero energy and $Z_{\text {nuc }}$ at infinite energy. For an ionized plasma with average ionization $\mathrm{Z}^{*}$ then $\mathrm{f}_{1}=\mathrm{Z}^{*}$ at $\mathrm{E}=0$.

In the absence of any bound electrons $f_{1}$ is equivalent to the number of free electrons per ion. Table 1 shows our best calculation of the partial components and total $\mathrm{f}_{1}$ value for each ionization stage of $\mathrm{Al}$ for a $14.7 \mathrm{~nm}(84.46 \mathrm{eV}) \mathrm{X}$ ray as used in the LLNL experiments. Taking the ratio of $\mathrm{f} 1$ to the number of free electrons in Table 1 gives the ratio of the measured electron density to the actual electron density. When the ratio is negative, the index of refraction is greater than one and the fringes bend the opposite direction than expected in the interferometer. The results of this calculation for $\mathrm{Al}$ are described in Ref. 9. This analysis was tedious, only included the ground state of each ionization stage, and did not include a distribution of ionization stages as one has in real plasma. For this reason we realized that the ability to calculate the index of refraction over a range of wavelength for any plasma condition would be a valuable tool for analyzing experiments.

\section{NEW ANALYSIS TOOLS}

The INFERNO code [10] has been used for many years to calculate the ionization conditions and absorption spectrum of plasmas under a wide variety of conditions. This code uses the average-atom technique. For finite temperatures and densities, the INFERNO code calculates a statistical population for occupation of one-electron Dirac orbitals in the plasma. We use a non-relativistic version of INFERNO in this work to calculate bound and continuum orbitals and the corresponding self-consistent potential. By applying linear response theory we obtain an average-atom version of the Kubo-Greenwood equation [20,21] for the frequency-dependent conductivity of the plasma. The imaginary part of the complex dielectric function is proportional to the conductivity. The real part of the dielectric function can be found from its imaginary part using a Kramers-Kronig [19] dispersion relation. The details of the KuboGreenwood formula applied to the average-atom model are described in Ref . 11.

To validate the average-atom results we compare them against calculations done with the OPAL code [12-14]. The OPAL code was developed at the LLNL to compute opacities of low- to mid-Z elements. The calculations use a manybody expansion of the grand canonical partition function. The atomic data are obtained from a parametric potential 
Table 1. The optical constant $f_{1}$ for different isoelectronic stages of $\mathrm{Al}$ at a photon energy $84.46 \mathrm{eV}(14.7 \mathrm{~nm})$. The total value of $f_{1}$ for a particular ionization stage is the sum of the contributions from the lines and continuum of the bound electrons together with the number of free electrons $\left(Z^{*}\right)$.

$\begin{array}{lcccccc}\text { Ion } & \text { L3 edge }(\mathrm{eV}) & \text { Line } & \text { Continuum } & \text { Free } & \mathrm{f}_{1} \text { (total) } & \text { Ratio }\left(\mathrm{f}_{1} / \text { free }\right) \\ +0 & 73.1 & +2.61 & -3.46 & +0 & -0.85 & \\ +1 & 92.4 & -0.70 & -4.48 & +1 & -4.19 & -4.19 \\ +2 & 105.4 & -2.91 & -2.63 & +2 & -3.54 & -1.77 \\ +3 & 120.0 & -3.16 & -1.64 & +3 & -1.80 & -0.60 \\ +4 & 153.7 & -2.49 & -0.66 & +4 & +0.84 & 0.21 \\ +5 & 190.5 & -1.17 & -0.29 & +5 & +3.54 & 0.71 \\ +6 & 241.4 & -0.57 & -0.13 & +6 & +5.30 & 0.88 \\ +7 & 284.6 & -0.20 & -0.06 & +7 & +6.73 & 0.96 \\ +8 & 330.2 & +0.03 & -0.03 & +8 & +8.00 & 1.00 \\ +9 & 398.6 & +0.19 & -0.02 & +9 & +9.18 & 1.02\end{array}$

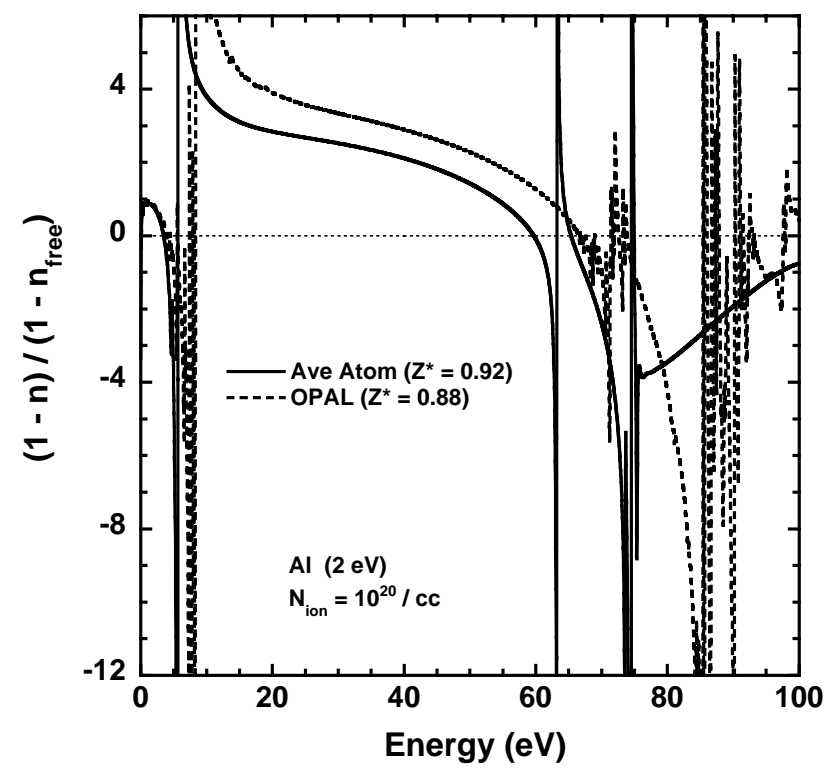

Fig. 1. Ratio (1-n) / (1-n free) versus photon energy for Al plasmas with temperatures of $2 \mathrm{eV}$. The solid curve is from the average atom code and the dashed line is from the OPAL code. The average ionization state is given by $Z^{*}$. The ion density is $10^{20} \mathrm{~cm}^{-3}$. The dotted line at a ratio of 0 is a visual aid. 
method that is fast enough for in-line calculations but whose accuracy is comparable to single configuration Dirac-Fock results. The calculations use detailed term accounting; for example, the bound-bound transitions are treated in full intermediate or pure LS coupling depending on the element. Degeneracy and plasma collective effects are included in inverse Bremsstrahlung and Thomson scattering. Most line broadening is treated with a Voigt profile that accounts for Doppler, natural width, and electron impacts. Linear Stark broadening by the ions is included for one-, two-, and threeelectron systems. In the OPAL code the plasma is treated as a mixture of atoms in discrete ionization states, thereby giving a more realistic description of individual lines and absorption edges. However the OPAL calculations can only be done for a limited range of ions.

\section{MODELING OF ALUMINUM PLASMAS}

In this section we utilize the average atom code to analyze Al plasmas and compare against OPAL calculations. To simplify the analysis, we assume the plasmas have a constant ion density of $10^{20} \mathrm{~cm}^{-3}$ and we vary the temperature to change the ionization state of the plasma. Since the fringe shifts are proportional to $f_{1}$ or $(1-n)$ we compare the ratio of $(1-n) /\left(1-n_{\text {free }}\right)$ where $n_{\text {free }}=1-\left(\mathrm{N}_{\text {elec }} / 2 \mathrm{~N}_{\text {crit }}\right)$ is the index of refraction due only to the free electron contribution. This ratio equals one if only the free electrons contribute to the index of refraction. This ratio also represents the ratio of the measured electron density to the actual electron density when one analyzes the interferometer experiment the traditional way assuming only free electrons contribute to the index of refraction.

Even though the calculations are done for a single ion density the ratio normalizes away the actual density so we expect the analysis to be valid over a wide range of densities. Both codes assume the electron, ion, and radiation temperatures are equal and the plasma is in local thermodynamic equilibrium (LTE). For a dynamic plasma that is not in equilibrium, the ionization condition $\left(Z^{*}\right)$ is a good way to compare plasma rather than the actual temperature of the plasma. $Z^{*}=1$ means the average ion is singly ionized, 2 means double ionized, etc.

Figure 1 plots the ratio $(1-n) /\left(1-n_{\text {free }}\right)$ versus photon energy for an Al plasma with a temperature of $2 \mathrm{eV}$. The dotted line at 0 is meant to be a visual aid. The average atom code results are shown with the solid curve while the OPAL calculation uses the dashed line. The $Z^{*}=0.92$ and 0.88 for the two codes, respectively, which means that the average ion is almost singly ionized. The ratio is negative from $75 \mathrm{eV}$ to $100 \mathrm{eV}$ with typical values for the average atom code ranging from -1 to -4 . A negative ratio means the index of refraction of the plasma is greater than one. At the $84.46 \mathrm{eV}$ energy of the Pd X-ray laser $(14.68 \mathrm{~nm})$, the ratio is -2.8 for the average atom code and -11 for OPAL. In the analysis of $\mathrm{Al}$ done in Ref. 9 the ratio for singly ionized $\mathrm{Al}$ was -4.2 . In all three calculations, a Mach-Zehnder or Fresnel bi-mirror interferometer as used in Refs. 4 and 5, would observe the fringes bend the opposite direction than was expected and by a larger distance than would be expected for the electron density being measured. At lower energies, such as the $26.44 \mathrm{eV}(46.9 \mathrm{~nm})$ of the Ne-like Ar X-ray laser [6] that has been used for many interferometer experiments, the ratio is 2.6 for the average atom code and 3.5 for OPAL. An interferometer built at this wavelength would measure an electron density that was about 3 times larger than the actual density. It is amazing that the ratio differs significantly from one over most of the energy range. Even at $4.68 \mathrm{eV}$, which is the energy of the $4^{\text {th }}$ harmonic of the $1.06 \mu \mathrm{m} \mathrm{Nd}$ laser, the ratio is predicted to be -3.0 for the average atom code and -0.5 for OPAL. It is important to realize that an average atom calculation will not have the position of the absorption lines and edges accurate enough to use the ratio to quantitatively analyze the experiments without some normalization to experiments but it is an invaluable first step in understanding the validity of the experiments and approximating the potential corrections needed to understand the experiments. The OPAL calculations should have the energies closer to the actual values but each situation needs to be compared against measured line positions to determine more accurate values. Both codes also have uncertainties in the line widths of the many resonance lines that will affect the shape of the calculation of $1-\mathrm{n}$. 

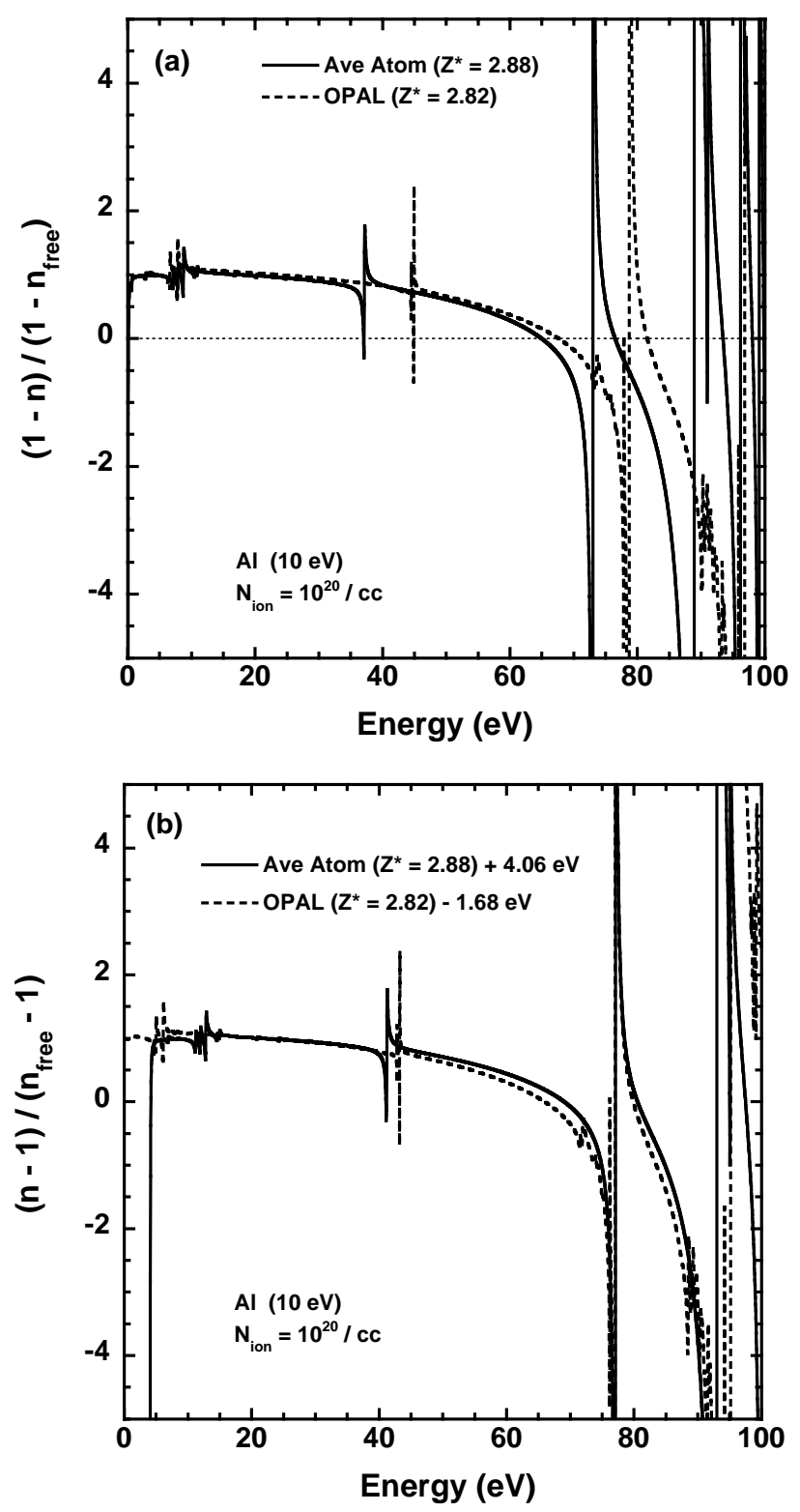

Fig. 2. Ratio (1-n) / (1-n free $)$ versus photon energy for Al plasma with temperature of $10 \mathrm{eV}$. The top figure (a) has the original calculated data while the bottom curve (b) has the energy scale shifted so that the calculated energies of the $2 p-3 s$ resonance line agrees with experimental value of $77.07 \mathrm{eV}$. The solid curve is from the average atom code and the dashed line is from the OPAL code. The average ionization state is given by $Z^{*}$. The ion density is $10^{20} \mathrm{~cm}^{-3}$. The dotted line at a ratio of 0 is a visual aid. 
Figure 2(a) plots the ratio for a $10 \mathrm{eV} \mathrm{Al}$ plasma with $\mathrm{Z}^{*}=2.88$ and 2.82 (almost triply ionized) for the average atom and OPAL codes, respectively. In triply ionized $\mathrm{Al}$ (Ne-like) the strong $2 \mathrm{p}-3 \mathrm{~s}$ resonance lines are centered at $77.07 \mathrm{eV}$ [17]. The average atom calculation shows a strong feature at $73.01 \mathrm{eV}$ due to these absorption lines while the OPAL results have the feature at $78.75 \mathrm{eV}$. In Fig. 2(a) the ratio at $84.46 \mathrm{eV}$ is -2.4 and -0.8 for the average atom and OPAL codes, respectively, If one shifts the energy axis by 4.06 and $-1.68 \mathrm{eV}$ for the average atom and OPAL codes to match the position of the Ne-like resonance line, then the ratio becomes -0.9 and -0.4 for the two codes, respectively. This is shown in Fig. 2(b). Ref. 9 predicted a ratio of -0.6 for triply ionized $\mathrm{Al}$ at this photon energy. All three analyses predict that the ratio is negative and that the index of refraction is greater than one. This is consistent with the fringe lines in an interferometer experiment bending the opposite direction than expected. The index of refraction of the $\mathrm{Al}$ plasma is predicted to be greater than one over a wide range of photon energies near $85 \mathrm{eV}$ and plasma conditions from neutral to triply ionized.

$\mathrm{X}$-rays need to be able to penetrate the $\mathrm{Al}$ plasma if they are to be used for interferometer measurements. This means the absorption needs to be low. Figure 3 shoes the absorption coefficient for an Al plasma at a temperature of 10 $\mathrm{eV}$ and an ion density of $10^{20} \mathrm{~cm}^{-3}$. For the region between 75 and $85 \mathrm{eV}$ and also between 40 and $70 \mathrm{eV}$ the average atom code predicts the absorption coefficient is less than $10 \mathrm{~cm}^{-1}$. Since a typical experiment $[4,5]$ uses a $0.1 \mathrm{~cm}$ long plasma, this means the plasma will be optically thin to the X-rays.

For more ionized Al plasma Fig, 4(a) shows the ratio $(1-n) /\left(1-n_{\text {free }}\right)$ versus photon energy for a $20 \mathrm{eV} \mathrm{Al}$ plasma with $Z^{*}=4.64$ and 4.46 for the average atom and OPAL codes, respectively. While the complicated structure is disappearing at energies near $84.46 \mathrm{eV}$ the ratio is still only 0.6 and 0.66 for the average atom and OPAL codes, respectively, and is in good agreement with the value of 0.5 that one interpolates from Ref. 9. Under these conditions the fringes would bend the expected direction in an interferometer experiment but the measured value of the electron density would be low by 30 to $50 \%$.

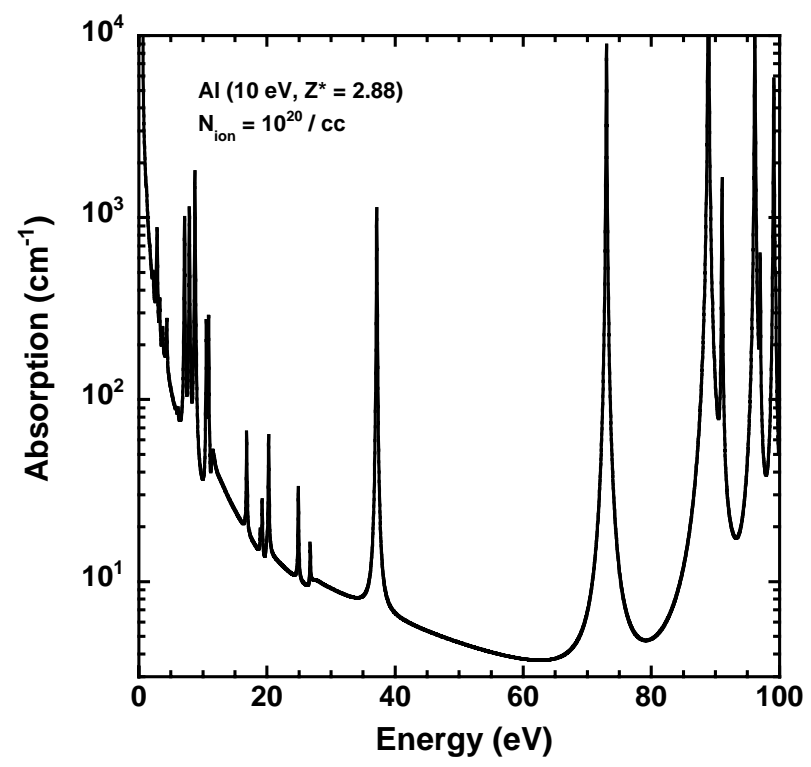

Fig. 3. Absorption coefficient versus photon energy for Al plasma with temperature of $10 \mathrm{eV}$. The ion density is $10^{20} \mathrm{~cm}^{-3}$. 

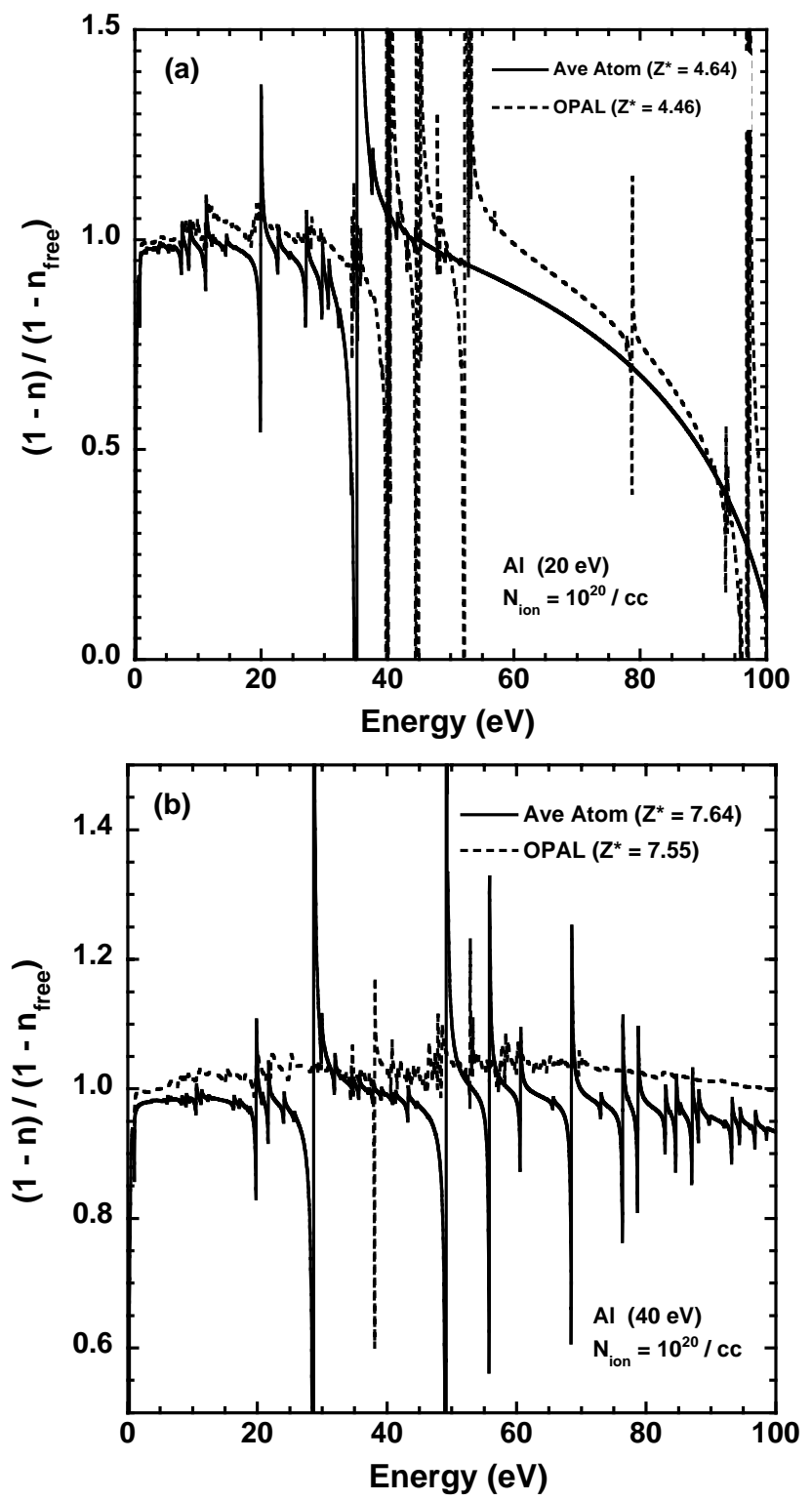

Fig. 4. Ratio (1-n) / (1- $\left.n_{\text {free }}\right)$ versus photon energy for Al plasmas with temperatures of (a) $20 \mathrm{eV}$ and (b) $40 \mathrm{eV}$. The solid curve is from the average atom code and the dashed line is from the OPAL code. The average ionization state is given by $Z^{*}$. The ion density is $10^{20} \mathrm{~cm}^{-3}$. 
For a $40 \mathrm{eV}$ Al plasma with $Z^{*}=7.64$ and 7.55 for the average atom and OPAL codes, respectively, Fig. 4(b) shows the ratio versus photon energy. The ratio is now within $10 \%$ of unity for most of the figure with the exception of some weak resonances. Al needs to be almost eight times ionized in order to approach the free electron approximation for the index of refraction. This is the same conclusion reached in Ref. 9. The Al experiments highlight how the traditional equation for the index of refraction in a plasma is not valid over a large range of plasma conditions and photon energies. At optical photon energies near a few $\mathrm{eV}$ the free electron approximation to the index for $\mathrm{Al}$ plasmas does appear to be valid except for the single ionized case. The average atom code and OPAL code give very similar values for $Z^{*}$. Both codes show similar trends for the ratio $(1-n) /\left(1-n_{\text {free }}\right)$ and there is good quantitative agreement when the energy scale is shifted to agree with known experimental lines or edges.

In Refs. 22 and 23 we have considered other plasmas such as carbon, titanium, and palladium. For all three plasmas the ratio $(1-n) /\left(1-n_{\text {free }}\right)$ is greater than one at many energies when the plasma are only a few times ionized. Fortunately as titanium is ionized to neon-like and palladium is ionized to nickel-like the ratio is very close to one at $84.46 \mathrm{eV}$ which indicates that the interferometer experiments to characterize the lasing plasma is in a valid regime for the free electron approximation.

\section{TIN AND XENON PLASMAS}

We have been looking for other plasmas in which we could observe a strong contribution from bound electrons that would make the index of refraction larger than one. For the interferometer that uses the Ne-like Ar X-ray laser at 46.9 $\mathrm{nm}(26.44 \mathrm{eV})$, doubly-ionized tin and silver look to be promising candidates to observe the negative fringe shifts. For the interferometer that uses the Ni-like Pd X-ray laser at $14.7 \mathrm{~nm}(84.46 \mathrm{eV})$, triply-ionized xenon looks very interesting. For these higher $\mathrm{Z}$ materials we have not yet used the average atom code to calculate them but we can estimate the index of refraction by doing some detailed modeling of the individual iso-electronic sequences.

Looking at $\mathrm{Sn}^{+2}$, we estimated the absorption coefficient by starting with the neutral absorption published in the Henke tables at higher energies and combining this with the measured photo-ionization edge at $30.3 \mathrm{eV}$, our multiconfiguration Dirac-Fock calculations for the low energy $5 \mathrm{~s}-5 \mathrm{p}$ lines near $9 \mathrm{eV}$, and the measured $4 \mathrm{~d}-5 \mathrm{p}$ lines near 27 $\mathrm{eV}$ [24]. Using the Kramers-Kronig dispersion relation we then determined the optical constant $\mathrm{fl}$ versus photon energy, as shown in Fig. 5. At the $26.44 \mathrm{eV}$ energy of the Ar X-ray laser line we estimate that $\mathrm{f} 1$ is -13 , which makes the index of refraction larger than one. The most important contribution to $\mathrm{fl}$ at this energy is from the $4 \mathrm{~d}-5 \mathrm{p}$ lines at 26.72 , 27.58, and $28.03 \mathrm{eV}$ that have been measured experimentally [24]. For neutral $\mathrm{Sn}$ we extrapolate the Henke data to estimate that $\mathrm{f1}$ is -1.79 at $26.44 \mathrm{eV}$. This suggests that experiments with $\mathrm{Sn}$ plasmas should observe fringe lines bending the opposite direction than expected due to the index of refraction being larger than one. More detailed modeling of the Sn plasma will be done in the future.

To find other prospective candidate materials we extrapolated the Henke data to estimate the f1 versus photon energy for neutral Mo, Pd, Ag, and Sb. Finding negative f1 values for neutral materials is usually a good clue to find negative $\mathrm{f} 1$ in plasma that are only a few times ionized. Figure 6 shows the estimated $\mathrm{fl}$ values versus photon energy for 5 materials. The other material with a negative $\mathrm{fl}$ value at $26.44 \mathrm{eV}$ is $\mathrm{Ag}$. We currently do not have sufficient experimental data to estimate the f1 value for Ag plasmas but based on calculations can speculate that doubly-ionized Ag will have $4 \mathrm{~d}-4 \mathrm{f}$ and $4 \mathrm{~d}-6 \mathrm{p}$ lines in the 25 to $30 \mathrm{eV}$ region that could result in a negative $\mathrm{fl}$ value at $26.44 \mathrm{eV}$. In addition the photo-ionization absorption edge for doubly-ionized $\mathrm{Ag}$ is at $35 \mathrm{eV}$ which means absorption in the $\mathrm{Ag}^{+2}$ plasma should be small for the $26.44 \mathrm{eV}$ energy of the Ar X-ray laser line. Looking at Fig. 6 one sees that Mo, Pd, and $\mathrm{Sb}$, have positive $\mathrm{fl}$ at $26.44 \mathrm{eV}$ even though Mo and $\mathrm{Pd}$ have negative values at other energies while $\mathrm{Sb}$ stays positive for the energies shown in the figure. Since the reflectivity of grazing incidence optics depends on f1 being positive an 
interesting experiment to verify the negative $\mathrm{f} 1$ values would be to see if the X-ray laser reflects off the material at grazing incidence. The X-ray laser should not reflect for any material with negative $\mathrm{fl}$.

For the interferometer at LLNL that uses the Pd X-ray laser at $14.7 \mathrm{~nm}(84.46 \mathrm{eV}), \mathrm{Xe}^{+3}$ looks very interesting. The Henke tables show neutral Xe with a value of -9 for $\mathrm{fl}$ at this energy. We used our atomic physics codes at LLNL together with the Henke data to construct the absorption spectrum for $\mathrm{Xe}^{+3}$. Recently Erik Emmons and his colleagues did a beautiful set of experiments [25] at the Advance Light Source (ALS) at Lawrence Berkeley Laboratory to measure the absorption coefficient of $\mathrm{Xe}^{+3}$ with high resolution $(0.05 \mathrm{eV})$ over the 37 to $117 \mathrm{eV}$ region. Emmons graciously provided us with their detailed data so that we could replace our calculated data with the measured data in the absorption spectrum we had constructed. Using the Kramers-Kronig dispersion relation we then calculated the optical constant $\mathrm{fl}$ from the absorption spectrum for $\mathrm{Xe}^{+3}$. This is shown in Fig. 7. At $84.46 \mathrm{eV}$, f1 is -35 , which means there should be a tremendous negative fringe shift with the index of refraction larger than one. From 72 to $87 \mathrm{eV} \mathrm{f1}$ is negative. This is primarily due to the very strong $4 \mathrm{~d}-4 \mathrm{f}$ line at $87.0 \mathrm{eV}$. For the Ni-like Ag X-ray laser that lases at $13.9 \mathrm{~nm}(89.25 \mathrm{eV})$ $\mathrm{f} 1$ is 16 since the laser line is on the high-energy side of the $\mathrm{Xe}^{+3}$ absorption line. In this case the experiment would bend the normal direction but the experiment would overestimate the electron density by a factor of five. We plan to do more calculations to understand Xe plasmas in the future.

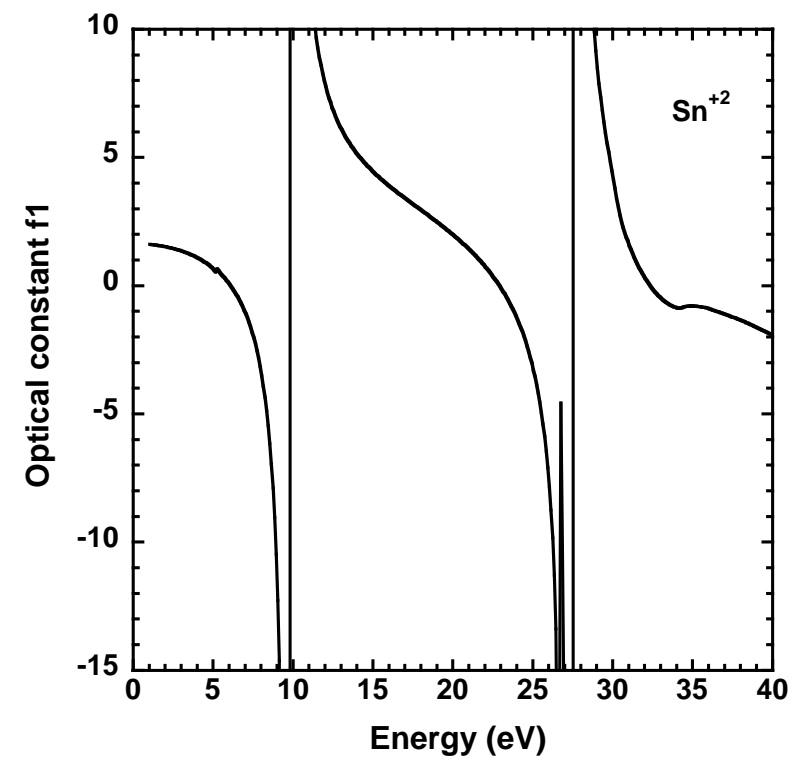

Fig. 5. Optical constant f1 versus photon energy for doubly-ionized tin. 


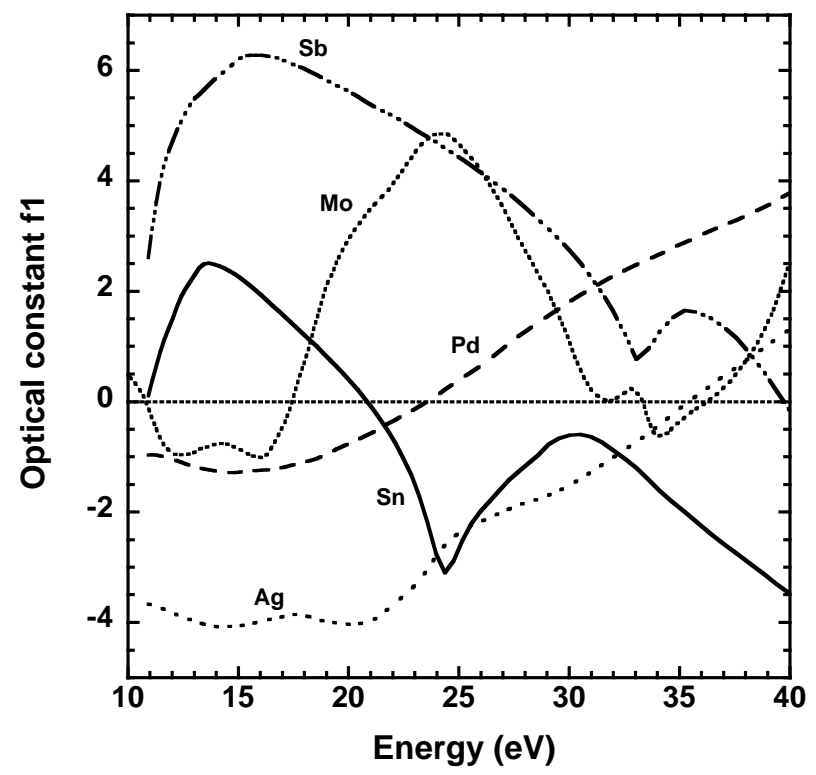

Fig. 6. Optical constant fl versus photon energy for neutral molybdenum, palladium, silver, tin, and antimony. These values are extrapolated from the Henke tables.

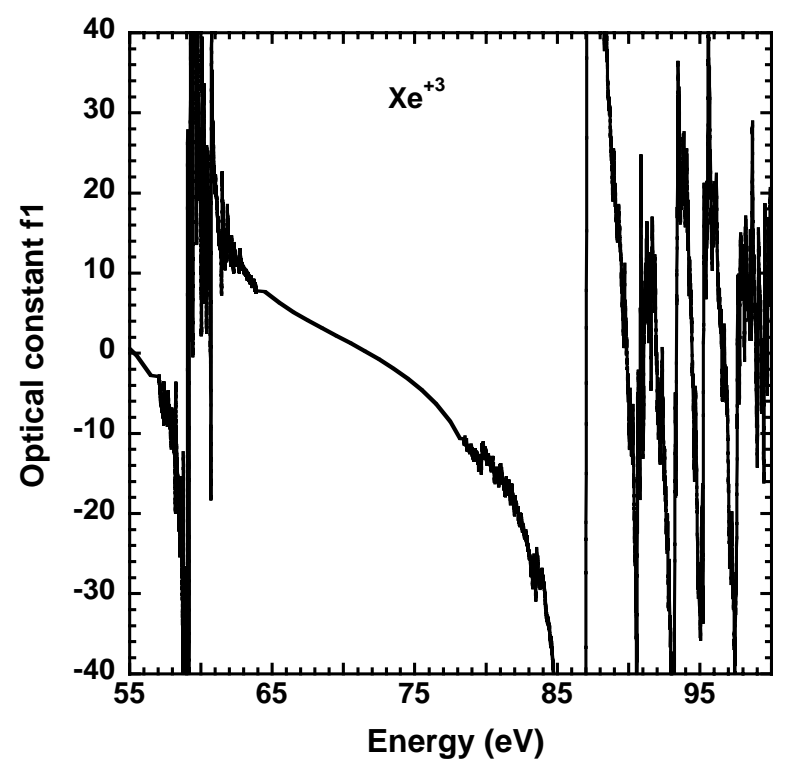

Fig. 7. Optical constant $\mathrm{fl}$ versus photon energy for triply-ionized xenon. 


\section{CONCLUSIONS}

For decades the analysis of plasma diagnostics such as interferometers have relied on the approximation that the index of refraction in plasmas is due solely to the free electrons. This makes the index of refraction less than one and is an essential assumption used in determining the critical density surface for energy deposition in the plasma and for doing photon transport calculations. Recent X-ray laser interferometer measurements of Al plasmas observed anomalous results with the index of refraction being greater than one. The analysis of the Al plasmas show that the anomalous dispersion from both the resonance lines and absorption edges due to the bound electrons can have the dominant contribution to the index of refraction over the photon range from the optical up to $100 \mathrm{eV}(12 \mathrm{~nm})$ soft X-rays. It is well known that a strong resonance line can cause anomalous results near the absorption line but this work shows that the effects from the resonance lines extend to photon energies located orders of magnitude further from the line centers than the corresponding line widths owing to the fact that they contribute through a dispersion integral. Similar long-range effects are shown for the absorption edges.

Utilizing a new average atom code we calculate the index of refraction in Al plasma and show many conditions over which the bound electron contribution dominates the free electrons as we explore photon energies from the optical to $100 \mathrm{eV}$ soft X-rays. The average-atom calculations are validated against the more detailed OPAL results.

We then search for other candidate materials that would have an index of refraction greater than one. We show that doubly-ionized tin should have an index greater than one at the energy of the Ne-like Ar X-ray laser at $46.9 \mathrm{~nm}$ (26.44 eV). Doubly-ionized silver also looks like a promising candidate at this energy. We discuss how one can use grazing incidence reflection to verify that materials do have an index greater than one in neutral materials. Finally we use recent high-resolution absorption measurements of triply-ionized xenon done at the ALS to show that this plasma will have an index greater than one and very large negative fringe shifts at the $84.46 \mathrm{eV}$ energy of the interferometer based on the Nilike Pd X-ray laser.

During the next decade X-ray free electron lasers and other sources will be available to probe a wider variety of plasmas at higher densities and shorter wavelengths so it will be even more essential to understand the index of refraction in plasmas. X-ray laser interferometers may become a valuable tool to measure the index of refraction of plasmas in the future.

\section{ACKNOWLEDGEMENTS}

We wish to thank Erik Emmons for providing us detailed absorption data from the $\mathrm{Xe}^{+3}$ measurements done at the ALS. This work was performed under the auspices of the U. S. Department of Energy by the University of California Lawrence Livermore National Laboratory under contract No.W-7405-ENG-48. This research was also sponsored by the National Nuclear Security Administration under the Stewardship Science Academic Alliances program through U. S. Department of Energy Research Grant \# DE-FG03-02NA00062. The work of one author (WRJ) was supported in part by NSF Grant No. PHY-0139928. 


\section{REFERENCES}

[1] G. J. Tallents, J. Phys. D. 17, 721 (1984).

[2] H. R. Griem, Principles of Plasma Spectroscopy, (Cambridge University Press, Cambridge, 1997) p. 9

[3] L. B. Da Silva, T. W. Barbee, Jr., R. Cauble, P. Celliers, D. Ciarlo, S. Libby, R. A. London, D. Matthews, S. Mrowka, J. C. Moreno, D. Ress, J. E. Trebes, A. S. Wan, and F. Weber, Phys. Rev. Lett. 74, 3991 (1995).

[4] H. Tang, O. Guilbaud, G. Jamelot, D. Ros, A. Klisnick, D. Joyeux, D. Phalippou, M. Kado, M. Nishikino, M. Kishimoto, K. Sukegawa, M. Ishino, K. Nagashima, and H. Daido, Appl. Phys. B 78, 975 (2004).

[5] J. Filevich, J. J. Rocca, M. C. Marconi, S. Moon, J. Nilsen, J. H. Scofield, J. Dunn, R. F. Smith, R. Keenan, J. R. Hunter, and V. N. Shlyaptsev, Phys. Rev. Lett. 94, 035005 (2005).

[6] J. Filevich, K. Kanizay, M. C. Marconi, J. L. A. Chilla, and J. J. Rocca, Opt. Lett 25, 356 (2000).

[7] D. Descamps, C. Lyngå, J. Norin, A. L'Hullier, C.-G. Wahlström, J.-F. Hergott, H. Merdji, P. Salières, M. Bellini, and T. W. Hänsch, Opt. Lett. 25, 135 (2000).

[8] A. Meseck, M. Abo-Bakr, D. Krämer, B. Kuske, and S. Reiche, Nucl. Inst. And Meth. A 528, 577 (2004).

[9] J. Nilsen and J. H. Scofield, Opt. Lett. 29, 2677 (2004).

[10] D. A. Liberman, JQSRT 27, 335 (1982).

[11] W. R. Johnson, C. Guet, and G. F. Bertsch "Optical properties of plasmas based on an average-atom model,“ J. Quant. Spectrosc. Radiat. Transfer (in press 2005).

[12] C. A. Iglesias, F. J. Rogers, and B. G. Wilson, Astrophys. J. Lett. 322, L45 (1987).

[13] F. J. Rogers, B. G. Wilson, and C. A. Iglesias, Phys. Rev. A 38, 5007 (1988).

[14] C. A. Iglesias and F. J. Rogers Astrophys. J. 464, 943 (1996).

[15] A. Aguilar, J. B. West, R. A. Phaneuf, R. L. Brooks, F. Folkmann, H. Kjeldsen, J. D. Bozek, A. S. Schlachter, and

C. Cisneros, Phys Rev A 67, 012701 (2003).

[16] J. B. West, J. Phys. B 34, R45 - R91 (2001).

[17] I. M. Savukov, J Phys B 36, 4789 - 4797, (2003).

[18] B. L. Henke, E. M. Gullikson, and J. C. Davis, ADNDT 54, 181 - 342 (1993).

[19] L. D. Landau and E. M. Lifshitz, Electrodynamics of Continuous Media, (Pergamon, New York, 1984) pp. 280 -

283

[20] D. A. Greenwood, Proc. Phys. Soc. London 715, 585 (1958).

[21] R. Kubo, J. Phys. Soc. Jpn. 12, 570 (1957).

[22] J. Nilsen, W. R. Johnson, C. A. Iglesias, and J. H. Scofield, "Impact of anomalous dispersion on the interferometer measurements of plasmas," J. Quant. Spectrosc. Radiat. Transfer (in press 2005). Also available as University of California report UCRL-JRNL-208675.

[23] J. Nilsen and W. R. Johnson, "Plasma interferometry and how the bound electron contribution can bend fringes in unexpected ways,” Appl. Opt. (in press 2005). Also available as University of California report UCRL-JRNL-209655.

[24] P. Dunne, F. O’Reilly, G. O’Sullivan, and N. Murphy, J. Phys. B 32, L597 (1999).

[25] E. D. Emmons, A. Aguilar, M. F. Gharaibeh, S. W. J. Scully, R. A. Phaneuf, A. L. D. Kilcoyne, A. S. Schlachter, I. Àlverez, C. Cisneros, and G. Hinojosa, Phys. Rev. A 71, 042704 (2005). 